\title{
Estudio palinológico preliminar en especies de Adesmia (Fabaceae) del noroeste de Argentina
}

\author{
Preliminary palynological study on Adesmia species (Fabaceae) \\ from northwestern Argentina
}

D.o.I.: doi.org/10.30550/j.lil/2018.55.1/4

\author{
Espeche María L. ${ }^{1}$; María E. García ${ }^{1}$; Nora J. F. Reyes ${ }^{1}$; María S. Caro ${ }^{2,3}$ \\ 1 Fundación Miguel Lillo, Laboratorio de Palinología. Miguel Lillo 251, (4000) San Miguel de Tucumán, \\ Argentina. \\ 2 Fundación Miguel Lillo, Instituto de Genética. \\ 3 Facultad de Ciencias Naturales e Instituto Miguel Lillo (U.N.T.) \\ Autor corresponsal: espechelau@yahoo.com.ar
} Resumen - Se analizó la morfología polínica de siete especies del género Adesmia (Faba-
ceae, Papilionoideae) que habitan la región montañosa del noroeste argentino: A. cordobensis
var. cordobensis Burkart, A. incana Vogel var. incana, A. muricata var. dentata (Lag.) Benth,
A. cytisoides Griseb., A. inflexa Griseb., A. trijuga Gillies ex Hook. \& Arn, A. schickendanzii
Griseb. Las muestras provienen de material fresco y fueron procesadas según las técnicas
convencionales para polen acetolizado y no acetolizado. Se tomaron fotos con microscopio
óptico y microscopio electrónico de barrido. Los granos son isopolares, radiosimétricos, tricol-
porados, microrreticulados, heterobrocados. En material acetolizado son de tamaño mediano,
prolatos a prolato-esferoidales. En material no acetolizado son pequeños, oblatos esferoidales,
colpos anchos a nivel del ecuador, con extremos agudos. Solo en $A$. schickendanzii el grano no
acetolizado es mediano, prolato, los colpos son angostos (4-5 $\mu$ m) en relación con las otras
especies y las endoaperturas son lolongadas y no se ven prominentes como en las demás.
Con microscopio electrónico de barrido se corrobora la presencia de margen liso o con es-
casas microperforaciones en A. cordobensis var. cordobensis, A. muricata var. dentata, A.
cytisoides y A. incana var. incana, densamente microperforado en $A$. trijuga, mientras que
A. inflexa carece de margen y se observa el borde irregular del colpo. En base a las obser-
vaciones realizadas se elaboró una clave para diferenciar las especies estudiadas. El análisis
estadístico permitió establecer las diferencias que apoyan la clasificación taxónómica de las
mismas. mía.

Palabras clave: Adesmia; Fabaceae; morfología polínica; noroeste de Argentina; taxono-

Abstract - The pollen morphology of seven species of the genus Adesmia (Fabaceae, Papilionoideae) that inhabit the mountainous region of northwest argentine was analyzed: $A$. cordobensis var cordobensis Burkart, A. incana Voguel var. incana, A. muricata var. dentata (Lag.) Benth, A. cytisoides Griseb., A. inflexa Griseb., A. trijuga Gillies ex Hook. \& Arn, A. schickendanzii Griseb. Samples were taken from fresh material and were processed according to conventional techniques for acetolyzed and non-acetolyzed pollen. Pictures were taken with

Ref. bibliográfica: Espeche, M. L., García, M. E., Reyes, N. J. F., Caro, M. S. (2018). Estudio palinológico preliminar en especies de Adesmia (Fabaceae) del noroeste de Argentina. Lilloa 55 (1): 37-48.

> Recibido: 28/08/17 - Aceptado: 11/04/18

$>$ URL de la revista: http://lilloa.lillo.org.ar

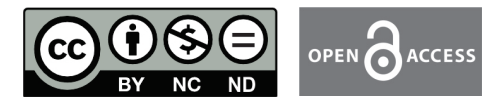

> Algunos derechos reservados. Esta obra está bajo una Licencia Creative Commons Atribución - No Comercial - Sin Obra Derivada 4.0 Internacional. 
an optical microscope and a scanning electron microscope. These grains are isopolar, radially symmetric, tricolporate, microreticulate and heterobrochate. In acetolyzed material they are of medium size, prolate to prolate-spheroidal. In non-acetolyzed material they are small, oblate spheroidal with wide colpi in the equatorial plane, and sharp ends. Only in the case of $A$. schickendanzii the non-acetolyzed grain is medium size, prolate, with narrow colpi (4-5 $\mu \mathrm{m}$ ) in relation to other species, and the endoapertures are lolongate, but not as prominent as in the others. With scanning electron microscope the presence of smooth margin or with few microperforations in A. cordobensis, var. cordobensis, A. muricata var. dentata, A. cytisoides y $A$. incana var. incana, $A$. cytisoides and $A$. incana is confirmed. In $A$. trijuga margin is densely microperforated, while $A$. inflexa lacks of margin and the irregular border of the colpi is observed. On the basis of the observations made, a key was developed to identify the species studied. The statistical analysis allowed to establish the differences that support their taxonomic classification.

Keywords: Adesmia; Fabaceae; northwest of Argentina; pollen morphology; taxonomy.

\section{INTRODUCCIÓN}

En América del sur, el género Adesmia DC. (Fabaceae, Papilionoideae) es el más amplio de las Leguminosas, con aproximadamente 240 especies. La mayor diversidad específica se encuentra en la cordillera chileno-argentina principalmente en áreas occidentales de las regiones montañosas semiáridas o áridas, en altitudes que varían entre 900-4000 m snm. Forma parte de la flora autóctona argentina, con cerca de 100 especies, (Zuloaga, Morrone, Rodríguez, 1999; Ulibarri y Burkart, 2000), muchas de ellas se distribuyen en el noroeste, principalmente en las provincias fitogeográficas Prepuna y Altoandina pudiendo llegar algunas especies a las provincias Chaqueña y Yunga (Ulibarri, 1996; Ulibarri y Burkart, 2000).

El alto polimorfismo que presenta en sus caracteres vegetativos y reproductivos ha dado lugar a numerosas sinonimias de las especies, por lo que se considera como un género complejo. Burkart (1967) estableció dos subgéneros: Adesmia Burk. que comprende plantas inermes (herbáceas, arbustivas o cojines), y Acanthadesmia Burk., reconocido por su carácter espinoso, (subarbustos, arbustos, cojines y placas leñosas), agrupando a su vez a las especies afines en 45 series (Ulibarri y Burkart, 2000).

Ambos subgéneros se distribuyen ampliamente en las regiones montañosas áridas y semiáridas del noroeste argentino (NOA), presentando el subgénero Acanthadesmia un marcado xeromorfismo (Ulibarri y Burkart, 2000). En su mayoría las especies de Ades- mia son forrajeras naturales; las espinosas son forrajeras nutritivas para las cabras y utilizadas como combustible por los lugareños (Burkart, 1952).

Los antecedentes palinológicos para el género son escasos y se limitan a los trabajos realizados por Heusser (1971) en A. arborea; Pire (1974) en A. echinus, A. uspallatensis, A. muricata var. muricata y A. muricata var. dentata; Markgraf y D'Antoni (1978) en Adesmia cf. pinifolia, A. subterranea y $A$. trijuga; Wingenroth y Heusser (1984) en $A$. capitellata, A. corymbosa, A. gayana, A. remyana y A. subterranea.

El objetivo del presente trabajo es analizar la morfología del polen, acetolizado y no acetolizado, de siete especies de Adesmia presentes en el NOA, con el fin de aportar datos que permitan la delimitación de estos taxones, establecer sus relaciones y contribuir al conocimiento de la Flora Polínica de esa región.

\section{MATERIALES Y MÉTODOS}

De las 7 especies estudiadas en el presente trabajo, 3 pertenecen al subgénero Adesmia: Adesmia cordobensis Burkart var. cordobensis, A. incana Voguel var. incana y A. muricata (Jacq.) DC var. dentata (Lag.) Benth, y 4 están incluídas en el subgénero Acanthadesmia: Adesmia cytisoides Griseb., A. inflexa Griseb., A. trijuga Gillies ex Hook. \& Arn., A. schickendanzii Griseb. Los ejemplares fueron recolectados en distintas localidades del Noroeste Argentino, en las provincias de 
Catamarca, Jujuy, Salta y Tucumán. El material de referencia fue depositado en el Herbario Fanerogámico de la Fundación Miguel Lillo (LIL). Las muestras palinológicas se incorporaron a la Palinoteca del Laboratorio de Palinología de la Fundación Miguel Lillo (PAL-TUC).

Se trabajó con material fresco recolectado. Para el estudio al microscopio óptico (MO) las muestras fueron procesadas mediante la técnica de Wodehouse (1935) para polen no acetolizado y acetolizadas mediante la técnica de Erdtman (1960). Las fotografías al MO se realizaron en el Laboratorio de Palinología de la Fundación Miguel Lillo con una cámara digital Canon Power Shot A 620 de 7,1 megapixeles. Para la observación en microscopio electrónico de barrido (MEB), los granos fueron metalizados con un baño de oro y se utilizó el equipo Zeiss Supra 55VP del Centro Integral de Microscopía Electrónica (CIME), Tucumán, del Sistema Nacional de Microscopía (SNM). Se aplicó la metodología tradicional para el análisis de la morfología de los granos de polen. Sobre un mínimo de 30 granos por especie se midieron los siguientes parámetros: eje polar (P), diámetro ecuatorial $(\mathrm{E})$, relación $\mathrm{P} / \mathrm{E}$, forma, posición y número de las aberturas, ancho y largo del colpo y del os, mesocolpio (M), apocolpio (Ap), estructura y escultura de la exina. La terminología empleada en las descripciones de los tipos polínicos corresponde a Punt, Blackmore, Nilsson, Le Thomas (1994) y Pire, Anzótegui, Cuadrado (1998). El tamaño de los granos de polen se determinó en base a la clasificación establecida por Erdtman (1952).

Analisis estadístico: Mediante el empleo del paquete estadístico InfoStat versión 2016 (Di Rienzo et al., 2016) se realizó un Análisis de Componentes Principales (ACP) para conocer la estructura de las relaciones entre las variables observadas y las especies estudiadas. La matriz se elaboró con variables obtenidas a partir de material acetolizado: $\mathrm{P}$ (eje polar), E (diámetro ecuatorial), P/E, AP (apocolpio), M (mesocolpio), LC (largo del colpo), AC (ancho del colpo), AO (ancho del ora), LO (largo del ora). Los resultados se presentan en un gráfico de ordenamiento, donde los taxones estudiados se representan con puntos y las variables con vectores dentro de un sistema cartesiano.

\section{MATERIAL ESTUDIADO}

Adesmia cordobensis Burkart var. cordobensis: ARGENTINA. Prov. Catamarca, Dpto. Andalgalá, Cuesta Las Chilcas, 27³8'09"S 66011'28"O, 1156 m snm; 7-III-05, Caro 14 (LIL); 27037'94"S 6609'34" O, 1940 m snm; 02-III-2007, Caro 28 (LIL) (PAL-TUC 790).

Adesmia cytisoides Griseb: ARGENTINA. Prov. Catamarca, Dpto Andalgalá, Cuesta a Capillitas, 27029'18"S 66²2'45"O, $2714 \mathrm{~m}$ snm; 03-III-2007, Caro 31 (LIL) (PAL-TUC 786).

Adesmia incana Vogel var. incana: ARGENTINA. Prov. Tucumán. Dpto. Burruyacú, Alto de Medina, 2624'92,1"S 6503'22,7"O,1526 m snm, 04-II-2011, Caro $S / N$ (PAL-TUC 812).

Adesmia inflexa Griseb: ARGENTINA. Prov. Tucumán, Dpto. Tafí del Valle, Los Corpitos, 26038'27,84"S 6549'19,62"O, 2631 m snm; 18-II-2014, Caro S/N (LIL);26043'25,3”S 6547'53,3"O, 2934 m snm; 13-III-2016, Caro $S / N$ (LIL). (PAL-TUC 787).

Adesmia muricata (Jacq.) DC. var. dentata (Lag.) Benth.: ARGENTINA. Prov. Tucumán, Dpto. Tafí del Valle, El Rincón, 2657'21,7”S 654'06,2"O, 2186 m snm; 02-III-2010, Caro 59 (LIL) (PAL-TUC 785).

Adesmia schickendanzii Griseb: ARGENTINA. Prov. Jujuy, Dpto. Tumbaya, Cuesta del Lipan, 52,23 41'38,3"S 65'38'57'O, 4155 m snm, 17- II-2014; Caro 117 (LIL); Prov. Tucumán, Dpto. Tafí del Valle, Lara, 26²2 '63"S 6540'68,8”O, 3260 m snm 30-XI-2009, Caro 57 (LIL); 26²2'71.5”S 6540'82”O, 3306 m snm; 30-XI-2009 Caro 58 (LIL); 26²2' 42,8"S 65040'48,8"O, $3278 \mathrm{~m}$ snm, 04-IV2013; Caro $S / N$ (LIL) (PAL-TUC 788).

Adesmia trijuga Gillies ex Hook. \& Arn.: ARGENTINA, Prov. Tucumán, Dpto. Tafí del Valle, Los Corpitos, 26038'27"S 6549'19”O, 2631 m snm, 3-II-2001, Caro S/N (LIL); Prov. Salta, Dpto. Cachi, Cuesta del Obispo, 2509' '240"S 6543'179"'O, 1787 m snm, 1304-2010, Caro S/N (LIL) (PAL-TUC 789). 


\section{RESULTADOS}

DESCRIPCIÓN GENERAL

DE LOS GRANOS DE POLEN

MO (Fig.1) Polen acetolizado: Granos, isopolares, radiosimétricos, tricolporados. Prolato a prolato-esferoidales, ámbito circular a subtriangular. Colpos largos y angostos, constreñidos al nivel de las ora. Endoaperturas circulares a lolongadas, con anillo. Exina de 1 a 1,6 $\mu \mathrm{m}$ de de espesor, sexina igual a nexina. Engrosamiento de la nexina a nivel de endoaperturas formando costillas. Sexina microrreticulada.

Los granos son pequeños en $A$. cordobensis var. cordobensis (Fig.1 C y D), de 23-25 x 19-23 $\mu \mathrm{m}$, prolato-esferoidales y en $\mathrm{A}$. $\mathrm{mu}$ ricata var. dentata (Fig.1 G y $\mathrm{H}$ ), de 23-24 x 19-23 $\mu \mathrm{m}$, subprolatos. En vista ecuatorial presentan contorno rectangular. Los colpos se ven lineales, las endoaperturas lolongadas. En vista polar el contorno es circular, un poco deprimido en la zona apertural.

Los granos son medianos en el resto de las especies: en $A$. cytisoides (Fig. $1 \mathrm{~K} \mathrm{y} \mathrm{L)}$ de 30-34 x 26-29 $\mu \mathrm{m}$, subprolatos, endoaperturas lolongadas a circulares; en $A$ inflexa (Fig.1 S y T) de 25-26 x 21-27 $\mu \mathrm{m}$, prolato-esferoidales, endoaperturas circulares a lolongadas; en $A$. incana var. incana (Fig.1 O y P) de $26-27$ x 22-24 $\mu \mathrm{m}$, subprolatos, endoaperturas lolongadas; en A. trijuga (Fig.1 W y X) de $26-30$ x 22-26 $\mu$ m prolato-esferoidales, endoaperturas circulares y en $A$. schickendanzii (Fig.1 A y B ) de 26-30 x 20-23 $\mu \mathrm{m}$, prolatos, endoaperturas lolongadas. En vista ecuatorial $A$. cytisoides y $A$ inflexa son de contorno circular a ovalado, en $A$. incana var. incana y en $A$ trijuga son de contorno rectangular, en el caso de $A$. schickendanzii son marcadamente ovalados. En vista polar, los granos de estas 5 especies son circulares a subtriangulares, anguloaperturados.

Polen no acetolizado: En A. cordobensis var. cordobensis (Fig. 1 A y B), A. muricata var. dentata (Fig. 1 E y F), A. cytisoides (Fig. $1 \mathrm{I}$ y J), A. incana var. incana (Fig. $1 \mathrm{M} \mathrm{y}$ N), A. inflexa (Fig 1 Q y R) y A. trijuga (Fig. $1 \mathrm{U} \mathrm{y} \mathrm{V}$ ) los granos tienen ámbitos circulares a subtriangulares convexos, anguloaper- turados. Son oblato esferoidales y en vista ecuatorial se aprecian los colpos anchos a nivel del ecuador, con extremos agudos. Las endoaperturas son prominentes, circulares o levemente lolongadas, con anillo.

Solo en A. schickendanzii (Fig. 1 Y y Z) el grano no acetolizado es prolato, los colpos son angostos $(4-5 \mu \mathrm{m})$ y las endoaperturas son lolongadas y no se ven prominentes como en las otras especies.

En la Tabla 1 se detallan las medidas de los diferentes parámetros considerados en la descripción de la morfología polínica de los granos tratados con ambas técnicas.

MEB (Fig. 2). La superficie presenta un microretículo heterobrocado, el diámetro de los lúmenes, de forma variada, disminuye paulatinamente hacia el borde de los colpos. A excepción de $A$. inflexa (Fig. $2 \mathrm{M}-\mathrm{O}$ ), en la que se destaca el borde irregular del colpo, el resto de especies presenta un margen que puede variar desde densamente perforado con borde irregular como A. trijuga (Fig. 2 $\mathrm{P}-\mathrm{R})$, perforado con borde irregular como en $A$. cytisoides (Fig. 2 G-I) y A. incana var. incana (Fig. $2 \mathrm{~J}-\mathrm{L}$ ), escasamente perforado con borde regular como en $A$. cordobensis var. cordobensis (Fig. 2 A-C) a liso con borde regular como en $A$. muricata var. dentata (Fig. 2 D-F). La membrana apertural es lisa en $A$. muricata var. dentata, $A$. incana var. incana y $A$. trijuga; es rugosa en $A$. cytisoides; es granulada en $A$. cordobensis var. cordobensis y A. inflexa. En todas estas especies se presentan elementos de sexina en forma granular a lo largo de la zona central de los colpos. En A. schickendanzii los granos prolatos presentan los colpos muy plegados, por lo que no se observa margen ni membrana apertural (Fig.2 S y T).

En base a las características morfológicas del polen, se elaboró la siguiente clave para diferenciar las 7 especies estudiadas.

Clave DE IDENTIFICACIÓN DE ESPECIES DE ADESMIA ESTUDIADAS EN BASE A LA MORFOLOGÍA POLÍNICA

Granos de polen de tamaño pequeño ... 

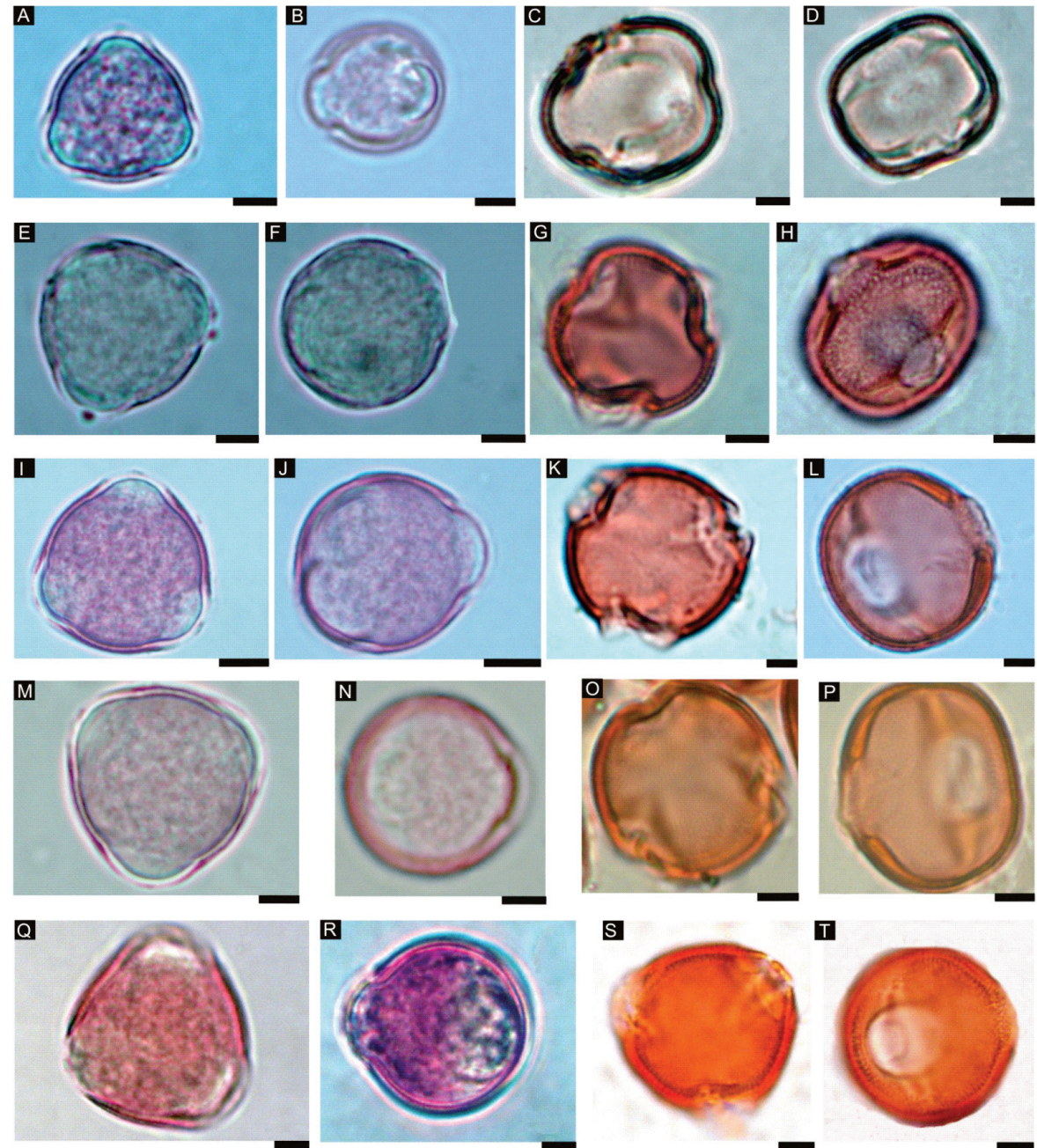

S

T
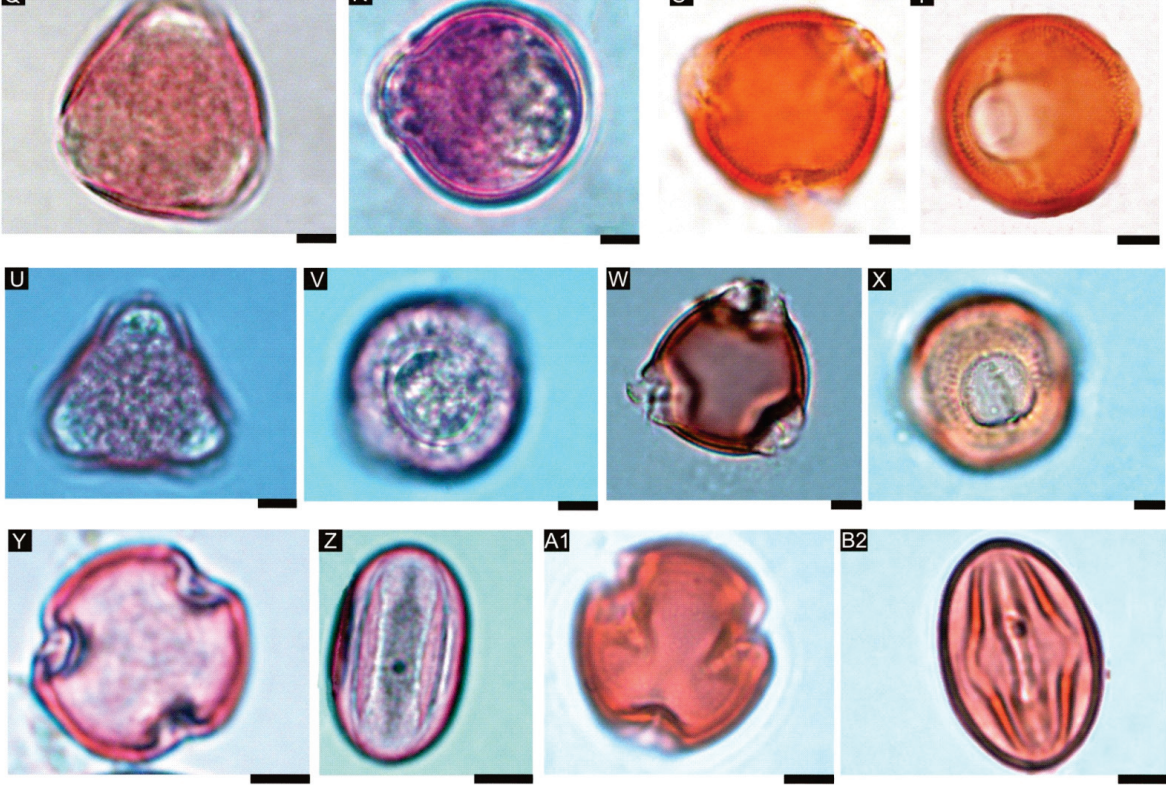

B2

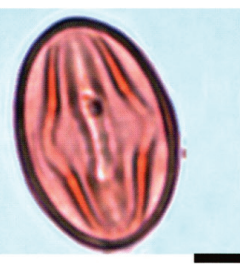

Fig. 1. MO. Para cada una de las especies se presentan 4 fotos dispuestas por fila correspondiendo las 2 primeras a material no acetolizado y las otras 2 a material acetolizado, en vista polar y ecuatorial respectivamente para cada caso. A-D) Adesmia cordobensis var. cordobensis. E-H] A. muricata var. dentata. I-L] A. cytisoides. M-P] A. incana var. incana. Q-T] A. inflexa. U-X] A. trijuga. $\left.\mathrm{Y}, \mathrm{Z}, \mathrm{A}_{1}, \mathrm{~B}_{1}\right] \mathrm{A}$. schickendanzii. Escala: $\mathrm{D}, \mathrm{H}$ y W: $1 \mu \mathrm{m} ; \mathrm{A}_{1} 2 \mu \mathrm{m}$; $B_{1} .0,5 \mu \mathrm{m}$. El resto $5 \mu \mathrm{m}$. Caro MS. S/N (LIL). 

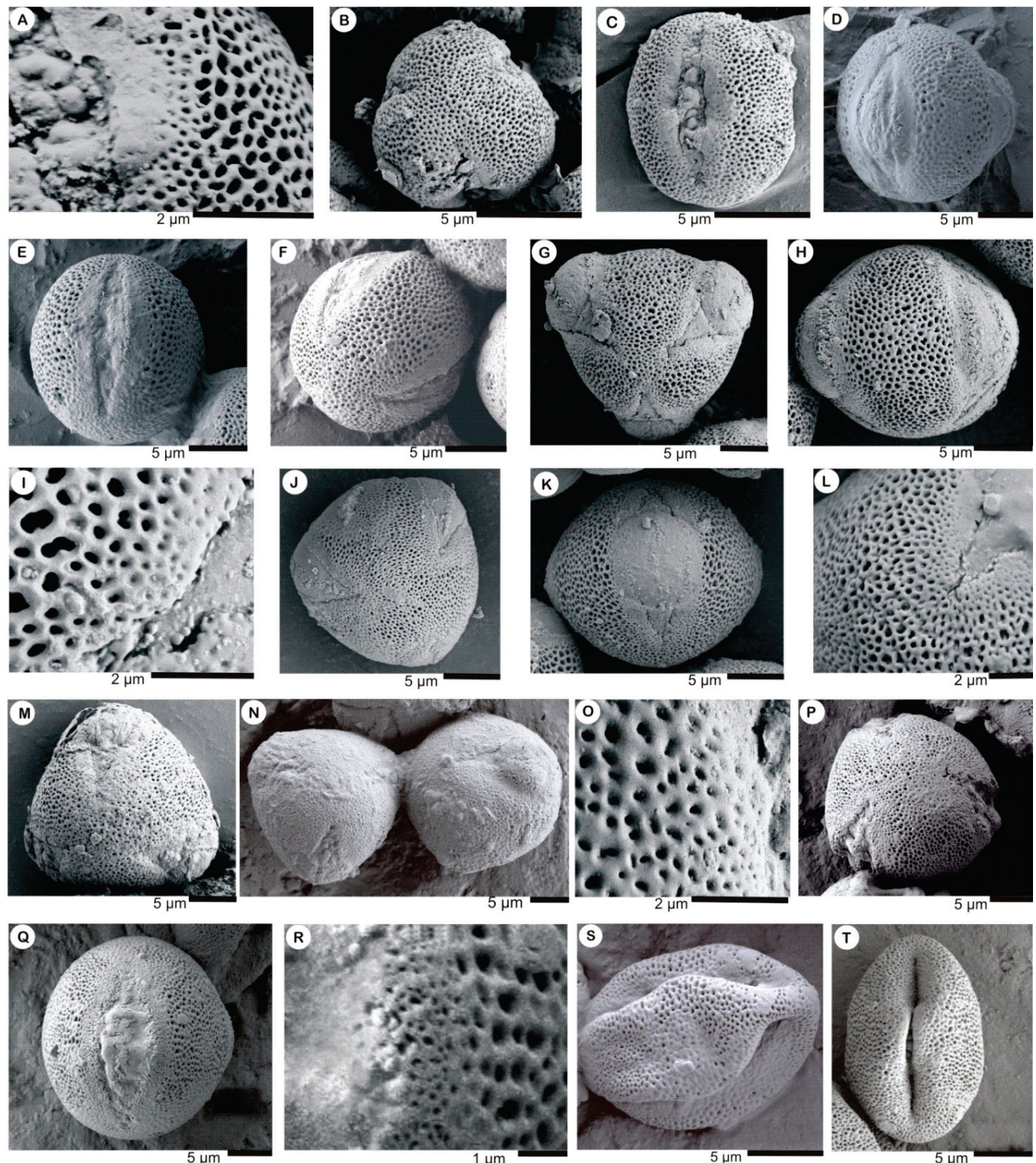

Fig. 2. MEB Adesmia cordobensis var. cordobensis. A] Detalle de superficie microrreticulada, margen con escasas perforaciones y membrana apertural granulada. B] Vista polar. C) Vista ecuatorial. A. muricata var. dentata. D y EJ Vista ecuatorial. FJ Vista subpolar. A. cytisoides. G) Vista polar. H) Vista ecuatorial. I) Detalle de superficie microrreticulada, margen con escasas perforaciones y membrana apertural lisa. A. incana var. incana, J) Vista polar. K] Vista ecuatorial. L) Detalle de superficie microrreticulada, margen con escasas perforaciones y membrana apertural lisa. A. inflexa. M] Vista polar. N) Vista polar y vista ecuatorial. 0) Detalle de superficie microrreticulada. A. trijuga. P) Vista polar. Q) Vista ecuatorial. R) Detalle de superficie microrreticulada y márgen densamente perforado. A. schickendanzii. S y T) Vista ecuatorial. Caro MS. S/N (LIL). 
Tabla 2. Análisis de Componentes Principales. Autovectores (e1 y e2) y correlaciones de los componentes principales [CP1 y CP2] con las variables.

\begin{tabular}{lccccc}
\hline \multirow{2}{*}{ Variables } & \multicolumn{2}{c}{ Autovectores } & & \multicolumn{2}{c}{ Correlaciones } \\
\cline { 2 - 3 } \cline { 5 - 6 } & $\mathrm{e} 1$ & $\mathrm{e} 2$ & & $\mathrm{CP1}$ & $\mathrm{CP2}$ \\
\hline $\mathrm{P}$ & 0,35 & 0,38 & & 0,8 & 0,51 \\
\hline $\mathrm{E}$ & 0,41 & $-0,13$ & & 0,95 & $-0,17$ \\
\hline $\mathrm{P} / \mathrm{E}$ & $-0,12$ & 0,62 & & $-0,29$ & 0,83 \\
\hline $\mathrm{AP}$ & 0,4 & $-0,19$ & & 0,93 & $-0,26$ \\
\hline $\mathrm{M}$ & 0,37 & 0,24 & & 0,86 & 0,33 \\
\hline $\mathrm{LC}$ & 0,35 & 0,05 & & 0,8 & 0,06 \\
\hline $\mathrm{AC}$ & 0,23 & 0,51 & & 0,53 & 0,68 \\
\hline $\mathrm{L} 0$ & 0,34 & $-0,18$ & & 0,79 & $-0,24$ \\
\hline $\mathrm{A} 0$ & 0,33 & $-0,27$ & & 0,75 & $-0,36$ \\
\hline
\end{tabular}

1' Granos de polen de tamaño mediano

2 Margen liso, membrana apertural lisa A. muricata

2' Margen con escasas perforaciones, membrana apertural granular Granos prolatos.......... A. cordobensis

A. schickendanzii

3' Granos prolato esferoidales a subprolato

4 Colpos sin márgen .............. A. inflexa

4' Colpos con margen .................. 5

5 Margen densamente perforado ..............

5' Margen escasamente perforado ...... 6
6 Granos de $26-27 \times 22-24 \mu \mathrm{m}$

6' Granos de 30-34 x 26-29 $\mu \mathrm{m}$

A. incana

A. cytisoides

\section{ANALISIS ESTADÍSTICO}

En la Figura 3 se observa el ordenamiento de las especies en relación a las variables analizadas. Se seleccionaron dos ejes o componentes principales para la interpretación de los resultados que en conjunto explicaron un $79 \%$ de la varianza total, el eje 1 o CP1 explicó un 59 \% y el eje 2 o CP2 un 19,9\%. En la Tabla 2 puede observarse que el CP1

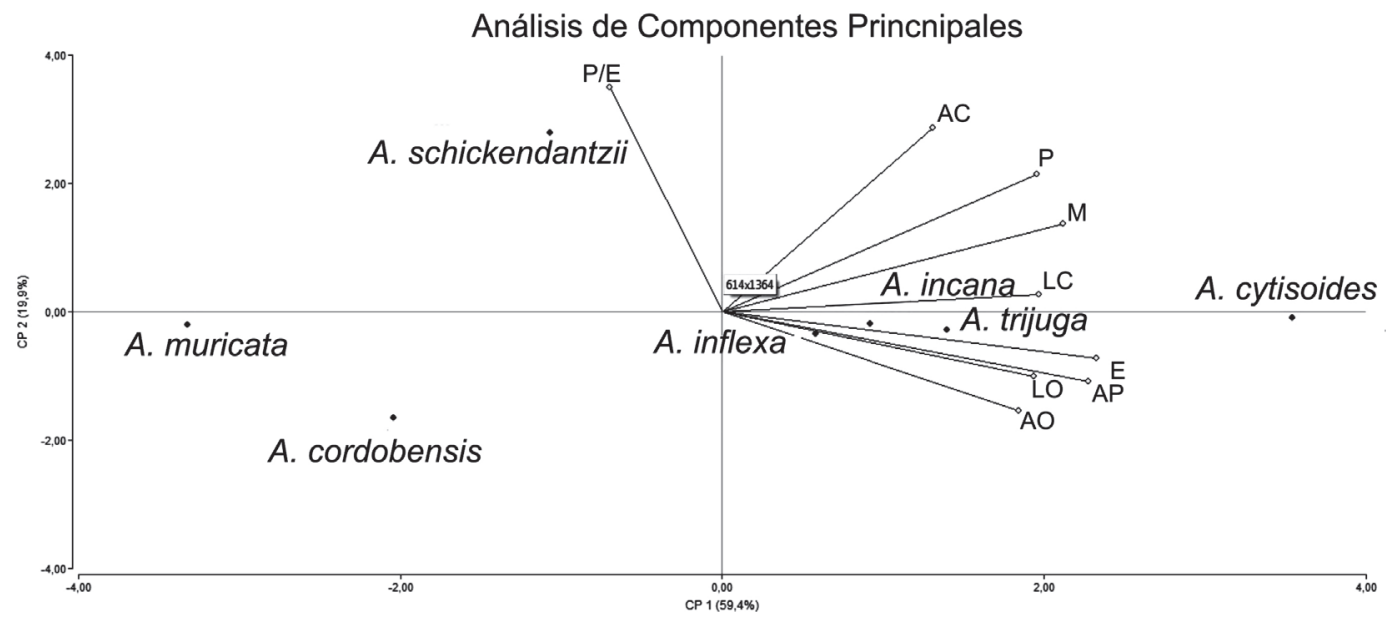

Figura 3. Ordenamiento de las especies en relación a las variables morfométricas de los granos de polen. P: eje polar, E: diámetro ecuatorial, P/E: relación eje polar / diámetro ecuatorial, AP: apocolpio, M: mesocolpio, LC: largo del colpo, AC: ancho del colpo, LO: largo del ora, AO: ancho del ora. 


\begin{tabular}{|c|c|c|c|c|c|c|c|c|c|c|c|c|c|c|}
\hline 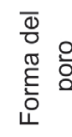 & 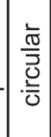 & 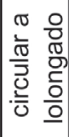 & \begin{tabular}{|l|}
$\frac{\frac{\pi}{0}}{\bar{J}}$ \\
$\frac{0}{0}$ \\
\end{tabular} & $\begin{array}{l}\text { 웜 } \\
\frac{\pi}{0} \\
\frac{1}{0} \\
\text { 응 }\end{array}$ & 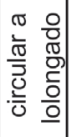 & 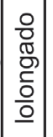 & 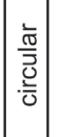 & 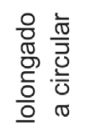 & \begin{tabular}{|l|}
$\frac{1}{0}$ \\
$\frac{\pi}{\bar{J}}$ \\
.$\frac{\bar{U}}{0}$
\end{tabular} & 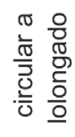 & \begin{tabular}{|c|} 
음 \\
$\mathbb{0}$ \\
$\stackrel{5}{5}$ \\
응 \\
\end{tabular} & $\begin{array}{l}\text { 음 } \\
\mathbb{\pi} \\
\stackrel{0}{0} \\
\text { 응 }\end{array}$ & & 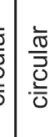 \\
\hline 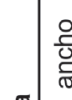 & $\frac{0}{1}$ & $\begin{array}{l}\stackrel{n}{0} \\
\hat{0} \\
\hat{6}\end{array}$ & \begin{tabular}{|l|}
0 \\
1 \\
\end{tabular} & $\begin{array}{l}\varphi \\
⿱ \\
1 \\
1\end{array} \mid$ & i & 穴 & $\frac{m}{\frac{m}{!}}$ & $\begin{array}{l}\text { L } \\
\stackrel{0}{\circ}\end{array}$ & 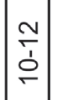 & م & 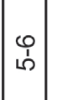 & n & $\frac{\stackrel{N}{\underline{T}}}{\Gamma}$ & $=\bar{\sigma}$ \\
\hline 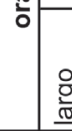 & $\bar{\sigma}$ & $\begin{array}{l}\stackrel{n}{N} \\
\stackrel{1}{0}\end{array}$ & \begin{tabular}{|l|}
$\infty$ \\
1 \\
1
\end{tabular} & ơ & $\underset{\infty}{\bar{a}}$ & $\mid \begin{array}{c}\mp \\
\bar{L} \\
0 \\
0 \\
0\end{array}$ & $\frac{m}{!}$ & $\stackrel{m}{\sim}$ & $\frac{m}{\grave{c}}$ & $\begin{array}{l}L^{\circ} \\
\infty\end{array}$ & $\frac{\stackrel{T}{I}}{\check{I}}$ & 웅 & $\frac{\stackrel{N}{I}}{\check{I}}$ & $\bar{\sigma}$ \\
\hline \begin{tabular}{l|l} 
& \multicolumn{2}{c}{} \\
& \multicolumn{2}{c}{} \\
& \\
\end{tabular} & $\frac{0}{6}$ & $\stackrel{L q}{+}$ & $\left|\begin{array}{l}0 \\
1 \\
1\end{array}\right|$ & $\stackrel{m}{\sim}$ & $\sigma$ & 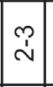 & \begin{tabular}{|l}
$\infty$ \\
1 \\
1
\end{tabular} & गै & 응 & $\stackrel{m}{\sim}$ & 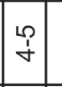 & p̊ & & 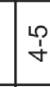 \\
\hline 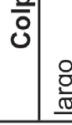 & $\stackrel{\infty}{\stackrel{2}{c}}$ & 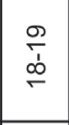 & \begin{tabular}{|l}
$\frac{\Omega}{d}$ \\
$\dot{z}$ \\
$\tau$
\end{tabular} & $\begin{array}{l}\bar{N} \\
\stackrel{\sigma}{\sigma}\end{array}$ & 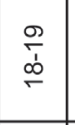 & 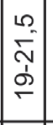 & $\approx$ & $\begin{array}{l}\stackrel{\infty}{N} \\
\stackrel{\omega}{N}\end{array}$ & $\mathbb{N}$ & ิㅗ & 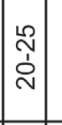 & స̃ & & নิ \\
\hline ய & - & 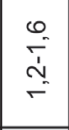 & $\mid \begin{array}{l}\text { 음 } \\
- \\
\dot{1} \\
\stackrel{1}{2} \\
0 \\
\end{array}$ & $\begin{array}{l}\sim \\
\sim \\
\stackrel{N}{L} \\
-\end{array}$ & $\stackrel{ \pm}{I}$ & 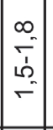 & 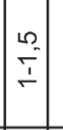 & $\stackrel{\Upsilon}{\check{T}}$ & $\stackrel{ }{=}$ & 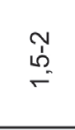 & $\mid \begin{array}{l}n \\
\\
\end{array}$ & 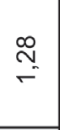 & $\sim$ & $N$ \\
\hline$\sum_{\Sigma}^{0}$ & $\frac{0}{\frac{O}{1}}$ & $\stackrel{\frac{N}{I}}{\leftarrow}$ & $\mid \begin{array}{l}O \\
\grave{1} \\
0\end{array}$ & 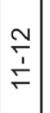 & 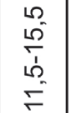 & 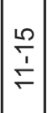 & $\stackrel{\widehat{\partial}}{\hat{\sigma}}$ & 혼 & 常 & $\stackrel{\sim}{\stackrel{i}{N}}$ & $\frac{\stackrel{N}{1}}{\check{I}}$ & $\frac{0}{b}$ & 동 & $\stackrel{\nabla}{\stackrel{s}{c}}$ \\
\hline$\frac{2}{4}$ & $\underset{\Sigma}{\bar{N}}$ & $\begin{array}{l}\text { o } \\
\text { 1े }\end{array}$ & 音 & $\infty$ & $\stackrel{\text { P }}{\stackrel{9}{r}}$ & $\mid$\begin{tabular}{|c|}
$\mid 0$ \\
0 \\
0 \\
$\infty$ \\
$\infty$
\end{tabular} & 免 & $\stackrel{\circ}{\infty}$ & 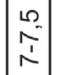 & $\begin{array}{l}\text { P } \\
\text { co }\end{array}$ & $\hat{o}$ & $\begin{array}{l}0 \\
1 \\
1 \\
\delta^{\circ}\end{array}$ & & \begin{tabular}{lll}
0 \\
\hdashline \\
\hdashline
\end{tabular} \\
\hline 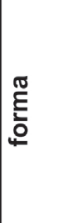 & \begin{tabular}{|l|}
$\bar{\pi}$ \\
$\frac{0}{0}$ \\
$\frac{\partial}{\omega}$ \\
$\frac{\omega}{\omega}$ \\
0 \\
0 \\
$\frac{0}{0}$ \\
$\frac{\pi}{0}$ \\
0
\end{tabular} & $\begin{array}{l}\frac{0}{0} \\
\frac{0}{0} \\
\frac{0}{0} \\
0 \\
0\end{array}$ & $\mid$\begin{tabular}{|c|}
$\bar{\sigma}$ \\
$\frac{0}{0}$ \\
$\frac{\partial}{\omega}$ \\
$\frac{\omega}{\omega}$ \\
0 \\
0 \\
$\frac{0}{0}$ \\
$\frac{0}{0}$ \\
\end{tabular} & 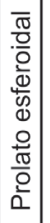 & $\begin{array}{l}\overline{\frac{\pi}{0}} \\
\frac{0}{0} \\
\frac{0}{0} \\
\frac{10}{y} \\
0 \\
\frac{0}{0} \\
\frac{\pi}{0}\end{array}$ & \begin{tabular}{|l|}
$\frac{0}{0}$ \\
$\frac{\pi}{0}$ \\
$\frac{2}{0}$ \\
$\frac{0}{3}$ \\
ம)
\end{tabular} & \begin{tabular}{|c|}
$\bar{\pi}$ \\
$\frac{0}{0}$ \\
$\overline{0}$ \\
$\frac{\omega}{\omega}$ \\
0 \\
0 \\
$\frac{0}{0}$ \\
$\frac{\pi}{0}$ \\
0
\end{tabular} & $\begin{array}{l}\frac{0}{\pi} \\
\frac{\pi}{0} \\
\frac{0}{0} \\
\frac{0}{3} \\
\text { क }\end{array}$ & $\mid$\begin{tabular}{|c|}
$\frac{\pi}{0}$ \\
$\frac{0}{0}$ \\
$\frac{\omega}{\omega}$ \\
$\frac{\omega}{\omega}$ \\
0 \\
$\frac{0}{0}$ \\
$\frac{0}{0}$
\end{tabular} & $\begin{array}{l}\overline{\bar{\pi}} \\
\frac{0}{0} \\
\overline{0} \\
\frac{0}{0} \\
0 \\
0 \\
0 \\
\frac{0}{0} \\
\frac{0}{0} \\
0\end{array}$ & 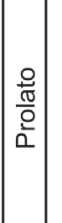 & $\begin{array}{l}\frac{0}{0} \\
\frac{\pi}{0} \\
\frac{0}{2}\end{array}$ & $\begin{array}{l}\bar{\sigma} \\
\frac{\pi}{0} \\
\frac{0}{\omega} \\
\frac{\omega}{\omega} \\
0 \\
0 \\
\frac{0}{0} \\
\frac{\pi}{0} \\
0\end{array}$ & 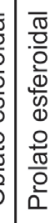 \\
\hline$\frac{w}{\alpha}$ & $\begin{array}{l}0 \\
0\end{array}$ & $\stackrel{\curvearrowright}{\check{C}}$ & $\mid$ & $\stackrel{m}{\leftarrow}$ & $\begin{array}{l}\text { \& } \\
0 \\
0\end{array}$ & $\stackrel{6}{\circ}$ & $\begin{array}{l}0 \\
0 \\
0\end{array}$ & $\stackrel{\infty}{\stackrel{一}{\leftarrow}}$ & \begin{tabular}{|l|}
$\infty$ \\
$\infty$ \\
0 \\
0
\end{tabular} & $\stackrel{\infty}{\stackrel{\infty}{-}}$ & $\underset{\sim}{\stackrel{g}{-}}$ & 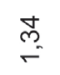 & ๙̃ & $\stackrel{m}{\longrightarrow}$ \\
\hline س & $\begin{array}{l}\bar{N} \\
\frac{1}{\delta} \\
o \\
\bar{N} \\
\bar{N}\end{array}$ & 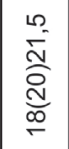 & 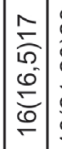 & 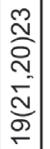 & $\begin{array}{l}\stackrel{J}{N} \\
\stackrel{N}{N} \\
\stackrel{N}{N}\end{array}$ & 竎 & $\begin{array}{l}\stackrel{\mathfrak{N}}{\mathrm{N}} \\
\stackrel{\mathrm{N}}{\mathrm{N}} \\
\mathrm{N}\end{array}$ & & 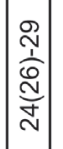 & 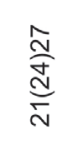 & $\mid \begin{array}{c}0 \\
\stackrel{0}{0} \\
\stackrel{0}{0} \\
\underline{0} \\
\underline{0}\end{array}$ & 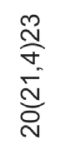 & 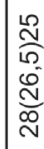 & 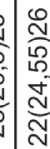 \\
\hline 0 & $\begin{array}{l}0 \\
\frac{0}{50} \\
00 \\
0 \\
0 \\
0\end{array}$ & 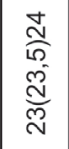 & 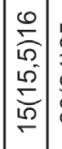 & 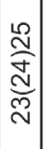 & $\begin{array}{l}\stackrel{2}{N} \\
\text { DN } \\
\text { N } \\
\text { N }\end{array}$ & 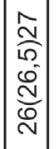 & 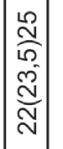 & 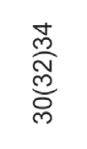 & $\stackrel{2}{N}$ & 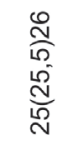 & 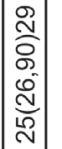 & 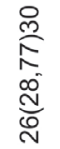 & 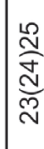 & 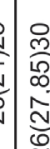 \\
\hline 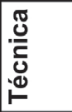 & $\S$ & $\varangle$ & $\mathbf{z}$ & $\varangle$ & 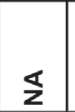 & $\varangle$ & $\mathbf{z}$ & $\varangle$ & $\mathbf{z}$ & $\varangle$ & $\mathbb{Z}$ & $\varangle$ & 乙 & 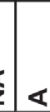 \\
\hline 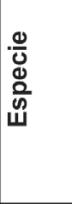 & & हैّ & 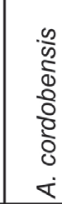 & & $\begin{array}{l}\mathbb{d} \\
\stackrel{.}{\subseteq} \\
<\end{array}$ & & & 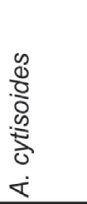 & & 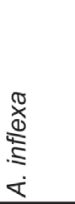 & $\frac{8}{8}$ & 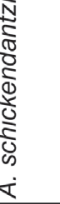 & & : \\
\hline
\end{tabular}


muestra altas correlaciones con la mayoría de las variables (E, AP, M, P, LC, LO, AO), E, AP y $M$ son las de mayor importancia en el eje separando a las especies por el tamaño de los granos y las aberturas.

En la Figura 3, respecto del CP1, se observa hacia la derecha a $A$. cytisioides que se diferencia por poseer granos de mayor tamaño, colpos largos y poros lolongados. A. cordobensis var. cordobensis y A. muricata var. dentata se ubican hacia la izquierda por debajo del eje horizontal, estas exhiben granos de menor tamaño, con colpos y poros de menor longitud y ancho. Por último A. incana var. incana, A. inflexa y A. trijuga conforman un grupo que exhibe características intermedias en relación a las especies descriptas anteriormente. Con respecto a el CP2 la variable de mayor relevancia fue P/ $\mathrm{E}$, que presenta un gradiente de concentración: hacia arriba del eje las especies (puntos) con mayor P/E y hacia abajo del mismo las especies con menor P/E. Esto implica un agrupamiento de las entidades por la forma de granos. A. schickendanzii posee el valor más alto y presenta granos prolatos; el valor más bajo se encuentra en $A$. cordobensis var. cordobensis la cual se caracteriza por poseer valores bajos de $\mathrm{P} / \mathrm{E}$ con granos de polen prolato-esferoidales a subprolatos. El resto de las especies se ubican cerca del eje horizontal que pasa por el cero, por lo que las diferencias de los P/E no son relevantes.

\section{DISCUSION}

En el NOA podemos encontrar representantes de los distintos tipos de hábitos del género Adesmia: herbáceas, subarbustos, arbustos y cojines.

A. cordobensis var. cordobensis, A. muricata var. dentata y $A$. incana var. incana, son plantas herbáceas, nativas que pertenecen al subgénero Adesmia, y se distribuyen en la provincia biogeográfica chaqueña.

A. cordobensis var. cordobensis y A. muricata var. dentata son las únicas especies de la serie Muricatae presentes en el noroeste argentino. A. cordobensis var. cordobensis es una especie endémica, crece entre rocas en la vegetación del monte o espinal entre los 900-2000m de altura (Ulibarri, 2008; Bianco, 2002), A. muricata var. dentata es típica de la región, crece en montes, en lecho seco de ríos y laderas pedregosas, entre los 900-3000 m snm (Burkart, 1954). Los granos de polen de estas 2 especies son los de menor tamaño de los 7 tipos estudiados, están comprendidos en el rango de pequeños en material no acetolizado, en las muestras acetolizadas aumentan un poco su tamaño. Las observaciones con MEB indican que no hay diferencias significativas entre ambas especies confirmando la ubicación taxonómica (serie Muricatae) establecida por Burkart (1967).

A. incana var. incana, pertenece a la serie Bicolor, crece en sierras semisecas hasta los 1800 m snm (Ulibarri y Burkart, 2000). Los granos de polen de esta especie son de tamaño mediano, lo que los diferencia de las dos mencionadas previamente para la serie Muricatae.

A cytisoides, A. inflexa, A. trijuga y A. schickendanzii son plantas espinosas comprendidas en el subgénero Acanthadesmia.

A. cytisoides y $A$. inflexa pertenecen a la serie Coluteoides y son especies endémicas de Argentina. En el noroeste habitan principalmente en las provincias biogeográficas Yungas y Prepuna. A. cytisoides es un arbusto que crece en faldeos orientales subhúmedos o semisecos entre los 2000-3000 m snm, a veces muy abundantes y con importancia fitogeográfica (Burkart, 1960; Ulibarri, 1996). Adesmia inflexa es un arbusto micrófilo; crece en laderas y cumbres entre los 1800-3700 m snm (Ulibarri y Burkart, 2000). Si bien estas 2 especies pertenecen a la misma serie (Coluteoides), desde el punto de vista palinológico se diferencian por el tamaño del grano, A. cytisoides (30-34 x 26-29 $\mu \mathrm{m}) \mathrm{y}$ en $A$. inflexa (25-26 x 21-27 $\mu \mathrm{m})$, además la primera presenta margen mientras que $A$. inflexa carece del mismo.

A. trijuga es una especie endémica y pertenece a la serie Mycrophyllae, que habita la provincia biogeográfica Altoandina. Es un arbusto que crece en suelos arenosos y pedregosos en quebradas húmedas o áridas 
entre los 1100-3000 m snm (Ulibarri, 1987; Ulibarri y Burkart, 2000). Palinológicamente se diferencia de las anteriores porque presenta un margen densamente perforado. La distribución geográfica de estas especies es similar pudiendo encontrarse juntas en una misma área.

A. schickendanzii, serie Subterráneae, es una especie nativa crio-xerófila, crece en grandes extensiones en la región andina del noroeste argentino; forma placas leñosas (cojines) en suelos arenosos y pedregosos. (Caro, Ruiz, Albornoz, 2016). El hábitat es diferente al resto, se encuentra en lugares elevados y ventosos entre los 2900 a $4000 \mathrm{~m}$. snm de las provincias biogeográficas Prepuneña (Catamarca, Tucumán, Salta) y Puneña (Jujuy) (Burkart, 1960; Ulibarri y Burkart, 2000). Es la única especie, del material estudiado, en la que los granos de polen tanto en material no acetolizado como en acetolizado son prolatos. Los colpos son angostos (4-5 $\mu \mathrm{m}$ ) en relación con las otras especies y las endoapeturas son lolongadas y no se ven prominentes como en los granos no acetolizados del resto de las especies. Las fotos con MEB permiten apreciar la superficie heterobrocada y la forma prolata de los granos, pero como los colpos están muy plegados, no se observa margen ni membrana apertural. Esta característica podría ser un aporte más a los diversos caracteres observados por Caro et al., (2016) que corroboran la adaptación de esta especie a ambientes xéricos.

Según la técnica aplicada para el estudio con MO, polen acetolizado y polen no acetolizado, permite observar variaciones en el tamaño y en la forma del grano de polen. En cuanto al tamaño, se destaca el material acetolizado un poco más grande que el no acetolizado. Respecto a la forma, los primeros pueden ser subprolatos, prolato-esferoidales hasta prolatos y en los segundos predominan los oblato-esferoidales.

El ACP se utilizo previamente para estudios en palinología (Ceballos, García, Slanis, Reyes, 2014; Mambrin, Avanza, Ferrucci, 2010). El análisis estadístico aplicado a las especies de Adesmia estudiadas corrobora las observaciones de la morfología polínica.
Pire (1974) analizó el polen de Adesmia echinus, A. uspallatensis, A. muricata var. muricata y A. muricata var. dentata, todas presentan granos medianos, más o menos esferoidales en vista ecuatorial y subtriangulares en vista polar, tricolporados, microrreticulados. Los detalles señalados por esta autora para A. muricata var. dentata son similares a los referidos en este trabajo.

La descripción de Adesmia cf. pinifolia, A. subterranea y A. trijuga, realizada por Markgraf y D'Antoni (1978) coincide con las especies estudiadas en este trabajo en cuanto al tamaño del grano, tipo y número de aberturas. En el caso de, A. trijuga, estos autores lo señalan como un grano esferoidal, sin embargo, las fotos que ilustran ese trabajo no reflejan la forma descripta, más bien serían granos oblato-esferoidales. Además, indican que los poros son lalongados, mientras que en nuestras observaciones son circulares.

Heusser (1971) describe Adesmia arborea con características similares a las mencionadas en este trabajo.

Wingenroth y Heusser (1984) estudian 5 especies de Adesmia (A. capitellata, A. corymbosa, A. gayana, A. remyana, A. subterranea) a las que describen con granos pequeños, tricolporados, pero ponen en duda la presencia de margen.

\section{CONCLUSIONES}

Se describe por primera vez la morfología de los granos de polen de $A$. cordobensis var. cordobensis, A. incana var. incana, A. cytisoides, A. inflexa y A. schickendanzii. En base a las observaciones realizadas se elaboró una clave para diferenciar las especies estudiadas. El análisis estadístico permitió establecer las diferencias que apoyan la clasificación establecida por Ulibarri y Burkart (2000); la serie Muricatae que incluye los taxones A. muricata var. dentata y A. cordobensis var. cordobensis con granos pequeños; A. incana var. incana (serie Bicolor) con granos medianos, $A$. cytisoides y $A$. inflexa (serie Coluteoides) con granos de tamaño variable, pero con características similares en la forma 
del polen. A. trijuga (serie Microphyllae) con granos medianos similares a las anteriores y con caracteres al MEB que permiten diferenciar claramente de $A$. inflexa lo que confirma que son especies diferentes (Ulibarri, 1985). A. schickendanzii (serie Subterraneae) con granos cuya morfología es diferente probablemente como una adaptación a ambientes xéricos.

Los resultados obtenidos nos inducen a una continuidad en estas investigaciones a fin de poder reconocer la amplitud en la diversidad de granos de polen de este género en las montañas del NO argentino.

Estos estudios significan un importante aporte a las distintas ramas de la palinología aplicada como la paleopalinología, la melisopalinología y la aeropalinología.

\section{AGRADECIMIENTOS}

Este estudio se llevó a cabo con el soporte financiero de la Fundación Miguel Lillo.

Las autoras agradecen a MSc. Lidia Benítez de Parra por su asesoramiento en el análisis estadístico de los datos.

\section{BIBLIOGRAFÍA}

Bianco, C. (2002). Growth forms, taxonomy, distribution, and uses of the Adesmia species (Leguminosae) in Central Argentina. J. Cramer. Berlin, Stuttgart.

Burkart, A. (1952). Las leguminosas argentinas silvestres y cultivadas. Acme Agency, SRL.

Burkart, A. (1954). Contribución al estudio del género Adesmia (Leguminosae) II. Darwiniana 10: 465-546.

Burkart, A. (1960). Contribución al estudio del género Adesmia (Leguminosae) III. Darwiniana 12 (1):81-136.

Burkart, A. (1967). Sinopsis del género sudamericano de Leguminosas Adesmia DC. Contribución al estudio del género Adesmia VII. Darwiniana 14: 463-568.

Caro, M. S., Ruiz, A. I. y Albornoz, P. L. (2016). Exomorfología, anatomía vegetativa y citología de Adesmia schickendanzii (Fabaceae-Papilionoideae) del Noroeste argentino. Lilloa 53 (1): 3-11.

Ceballos, S. J., García, M. E., Slanis, A. C. y Reyes, N. J. F. [2014). Aplicación de análisis de componentes principales en la identificación de subtipos polínicos en
Miconia (Melastomataceae). Lilloa 51 (2): 151-157.

Erdtman, G. (1952). Pollen morphology and plant taxonomy. Angiosperms (An introduction to Palynology I). Almqvist \& Wiksell, Stockholm

Erdtman, G. (1960). The acetolysis method. Svensk Botanisk Tidskrift 54: $561-$ 564.

Di Rienzo, J.A., Casanoves, F., Balzarini, M.G., Gonzalez, L., Tablada, M.y Robledo, C.W. (2016). Grupo InfoStat, FCA, Universidad Nacional de Córdoba, Argentina. www.infostat.com.ar Ultima consulta: junio 2017

Heusser, C. J., (1971). Pollen and spores of Chile. Univ. Arizona Press, Tucson.

Mambrín, M. V., Avanza, M. M. y Ferrucci, M. S. (2010). Análisis morfológico y morfométrico del polen de Corchorus, Heliocarpus, Luehea, Mollia y Triumfetta (Malvaceae, Grewioideae) en la región austral de América del sur. Darwiniana 48 (1): 45-58.

Markgraf, V. y D'Antoni, H. L. (1978). PolIen Flora of Argentina. Modern spore and pollen types of Pteridophyta, Gymnospermae and Angiospermae. University of Arizona Press, Tuxon.

Pire, S. M. (1974). Estudio palinológico de la tribu "Hedysareae" (Leguminosae). Bonplandia Tomo III No 12: 145-169.

Pire, M. S., Anzótegui, L. M. y Cuadrado, G. A. (1998). Flora Polínica del Nordeste Argentino volumen I Ed. Universidad Nacional del Nordeste

Punt, W., Blackmore, S., Nilsson, S. y Le Thomas, A. 1994. Glossary of pollen and spores terminology. Serie 1. LPP Contributions. LPP Foundation, Utrech.

Ulibarri, E A. (1985). Aclaración sobre el tipo de Adesmia inflexa Griseb y su sinonimia [Leguminosae-Papilionoideae). Darwiniana 26 (1-4): 285-287.

Ulibarri, E. A. (1987). Las especies de $A d$ esmia de la serie Microphyllae (Leguminosae-Papilionoideae). Darwiniana 27 (1-4): 315-388.

Ulibarri, E. A. (1996). Aportes Botánicos de Salta. Serie Flora. Flora del Valle de Lerma. Fabaceae 4: 1-11.

Ulibarri, E. A. y Burkart, A. (2000). Sinopsis de las especies de Adesmia de la Argentina. Darwiniana 38: 9-126.

Ulibarri, E. A. (2008). Adesmia (Fabaceae). En: F. O. Zuloaga, O. N. Morrone \& M. J. Belgrano [eds.].Catálogo de las Plantas Vasculares del Cono Sur. Missouri Botanical Garden Press, 2: 20862108 . 
Wingenroth, M. y Heusser, C. J. (1984). Polen en la alta cordillera: quebrada Benjamín Matienzo. Ed. Instituto Argentino de Nivología y Glaciología.

Wodehouse, R. P. (1935). Pollen grains. Mc Graw-Hill Book Company, Inc. New York and London.

Zuloaga, F. O., Morrone, O., Rodríguez, D. (1999). Análisis de la biodiversidad en plantas vasculares de la Argentina. Kurtziana 27 (1): 17-167. 